Arab Univ. J. Agric. Sci., Ain Shams Univ., Cairo, Egypt

28(4), 1167-1176, 2020

Website: http://ajs.journals.ekb.eg

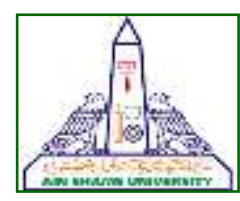

1167

\title{
Yield of Tomato Crop Irrigated with Untreated Industrial Sewage Effluent and Remediated with Potassium Silicate and Compost Applications
}

\author{
Rana HH Abo-Nofal ${ }^{1 \star}$, Eman A Diab ${ }^{1}$, Hashim M$^{2}$, Mohamed Z El-Shinawy², \\ Hany G Abd El-Gawad ${ }^{2}$
}

1- Environmental Pollution, Plant Ecology and Range Dept, Desert Research Center, Cairo, Egypt

2- Horticulture Dept, Fac of Agric, Ain Shams Univ, P.O. Box 68, Hadayek Shoubra 11241, Cairo, Egypt

*Corresponding author: ronyhosny65@gmail.com

Received 12 April, 2020

Accepted 10 September, 2020

\begin{abstract}
An experiment was conducted for the two successive seasons of 2017 and 2018 to investigate the deleterious effect of irrigating tomato plants with untreated industrial waste water and the possible ameliorating effects of compost and potassium silicate applications on the growth and production of the grown plants. Tomato seedlings of hybrid K186 were transplanted at the four-true leaf stage and irrigated with untreated waste effluent. Compost was applied during the soil preparation at rates of 0 (control), 10, 20, and $30 \mathrm{~m}^{3} / \mathrm{feddan}$. Potassium silicate was sprayed on the plants three times; at 20, 40 and 60 days after transplanting in the concentrations of 0 (control), 3, 4 and $5 \mathrm{~cm}^{3} / /$. Results revealed that vegetative growth and fruit yield of treated plants were increased by increasing compost and potassium silicate rates compared by the control (without compost and potassium silicate). The interaction effect of the treatment showed an added effect of both treatments on all measured parameters. Plant length, number of leaves and number of branches increased as the application rate of compost and potassium silicate increased. Similarly, SPAD readings showed similar positive and significant trend. In the contrary, contents of $\mathrm{Zn}, \mathrm{Pb}, \mathrm{Ni}, \mathrm{Cd}$ and Mnresponded negatively and significantly to the interaction effect of the treatments showing the highest effect with the treatment $5 \mathrm{~cm}^{3}$ potassium silicate associated with $30 \mathrm{~m}^{3}$ compost application compared to the control. Similar to the positive effect on vegetative growth, the positive additive effect of both treatments was clear on total fruit yield where the
\end{abstract}

highest effect was recorded with the treatment combining the highest rate of application of both potassium silicate and compost. It could be concluded that potassium silicate and compost applications can ameliorate the harmful effects of heavy metals in the soil.

Keywords: Tomato, Industrial waste water, Potassium silicate, Compost, Heavy metals, Yield

\section{Introduction}

Egypt was classified as an arid land with a very little amount of rain fall mainly in the north coast and having the Nile Rivera's the main source of irrigation water. Officials revealed that with the ongoing agricultural expansion and climate changes, Egypt is facing a serious shortage of fresh water supply forcing the country to recycle all available sources of water. Some growers in remote areas are using raw industrial sewage effluent to fulfill their crop water needs ignoring the possible dangerous of contamination of heavy metals of such water.

The negative effects of heavy metal on many aspects of agriculture have been reported. For example, heavy metal can be leached to the underground water (He et al 2004, Rattan et al 2005) causing the spread of pollution to other layers of the aquifers. Heavy metal polluted soil can negatively affect plant growth and production causing serious economic losses (Nagajyoti et al 2010). Moreover, Arao et al (2010) and Khan et al (2008) reported high health risks for people exposed to polluted agricultural soil. The problem of heavy metal pollution to the soil is 
more difficult compared to other types of pollution such organic contamination because they cannot be degraded by microorganisms and last in the soil for longer time.

The application of compost decreased heavy metal concentration in plants (Ramachandran and D'souza 1998). In this respect, Sharma and Dhaliwal (2019) found that decreased concentrations of toxic metals in soils with regular application of wastewater sewage sludge with compost treatments.

Some researchers used soil amendments to change the mobility and bioavailability of such heavy metals (Bolan et al 2014, Udeigwe et al 2011). One of such amendments was compost ( $\mathrm{Pa}$ radelo et al 2011). Compost is rich with mineral ions, humic substances, and microbes which influence the immobilization of heavy metals resulting in reduction of the ecological and environmental risk of heavy metals in agricultural soils (De la Fuente et al 2011, Udovic and McBride 2012). Adsorption, complexation, precipitation, and redox reactions may all be one or more process involving heavy metal immobilization (Huang et al 2010, Lagomarsino et al 2011, Park et al 2011, Vaca-Paulin et al 2006). Thus, in addition to the benefit of compost as an alternative for waste management, its application can reduce the harmful effects on the crop, lower economic losses, and decrease human health risks from heavy metals existing in the root zone. Beneficial effects of compost on the growth and production of some vegetable crops such as green beans were reported under different levels of irrigation (AbdelMawgoud 2005) as well as salinity (Abdel-Mawgoud et al 2010).

Another alternative to reduce heavy metals deteriorating effects on plants is the application of some nutritional and/or beneficial elements such as silicon (Emamverdian et al 2018). Epstein (1999) described silicon (Si) as a beneficial and possibly essential element for plants, which plays important roles in plant growth and development (Ma and Yamaji 2006, Gu 2012). Many researchers reported various evidence that the application of $\mathrm{Si}$ to soils can alleviate $\mathrm{Cd}$ or $\mathrm{Zn}$ toxicity in many plant species, including rice ( $\mathrm{Ma}$ et al 2015), maize (Liang et al 2005), wheat (Hussain et al 2015) and cotton (Farooq et al 2013).

Therefore, this work aims to investigate the effect of compost and potassium silicate applications, on the growth and production of tomato crop grown in soils irrigated with untreated industrial sewage effluent.

\section{Materials and Methods}

Seeds of tomato plant (Solanum lycopersicum L.) hybrid K186 were sown on $24^{\text {th }}$ and $28^{\text {th }}$ of April 2017 and 2018, respectively. After one month when the seedlings reached the fourth true leaf, they were transplanted in the open field in a sandy soil at a private farm in the area of Borg Al-Arab, Alexandria Governorate, Egypt. The soil physical and chemical analyses are shown in Table 1. Individual transplants were grown at the bottom of ridges $100 \mathrm{~cm}$ width and at $50 \mathrm{~cm}$ apart. Planting distances between plants are $50 \mathrm{~cm}$. The furrow irrigation was used and plants were irrigated using water from an industrial sewage channel. The chemical analysis of the irrigation water is shown in Table 1. Also, heavy metals analyses in soil and irrigation water during the two seasons of study are shown in Table 2.

Table 1. Physical and chemical analyses of soil and irrigation water during the two seasons of the study

\begin{tabular}{|c|c|c|}
\hline Soil properties & $\begin{array}{c}2017 \\
\text { season }\end{array}$ & $\begin{array}{c}2018 \\
\text { season }\end{array}$ \\
\hline \multicolumn{3}{|l|}{ I. Physical analysis } \\
\hline Sand $(\%)$ & 89.65 & 88.40 \\
\hline Silt (\%) & 6.12 & 6.15 \\
\hline Clay (\%) & 4.23 & 5.45 \\
\hline \multicolumn{3}{|l|}{ II. Chemical analysis } \\
\hline $\mathrm{pH}$ & 7.79 & 7.68 \\
\hline $\mathrm{EC}(\mathrm{dS} / \mathrm{m})$ & 8.73 & 8.61 \\
\hline $\mathrm{Ca}^{+2}(\mathrm{meq} / \mathrm{l})$ & 34.13 & 33.27 \\
\hline $\mathrm{Mg}^{+2}(\mathrm{meq} / \mathrm{l})$ & 18.02 & 18.10 \\
\hline $\mathrm{K}^{+}(\mathrm{meq} / \mathrm{l})$ & 2.96 & 3.08 \\
\hline $\mathrm{Na}^{+}(\mathrm{meq} / \mathrm{l})$ & 35.53 & 37.02 \\
\hline $\mathrm{Cl}^{-}(\mathrm{meq} / \mathrm{l})$ & 38.05 & 39.21 \\
\hline $\mathrm{HCO}_{3}^{-}(\mathrm{meq} / \mathrm{l})$ & 5.70 & 5.84 \\
\hline $\begin{array}{l}\text { Chemical analysis of } \\
\text { irrigation water }\end{array}$ & $\begin{array}{l}2017 \\
\text { season }\end{array}$ & $\begin{array}{l}2018 \\
\text { season }\end{array}$ \\
\hline \multirow{9}{*}{$\begin{array}{l}\mathrm{pH} \\
\mathrm{EC}(\mathrm{ppm}) \\
\mathrm{Ca}^{+2}(\mathrm{meq} / \mathrm{l}) \\
\mathrm{Mg}^{+2}(\mathrm{meq} / \mathrm{l}) \\
\mathrm{K}^{+}(\mathrm{meq} / \mathrm{l}) \\
\mathrm{Na}^{+}(\mathrm{meq} / \mathrm{l}) \\
\mathrm{Cl}^{-}(\text {meq/l) } \\
\mathrm{SO}_{4}^{-2}(\mathrm{meq} / \mathrm{l})\end{array}$} & & \\
\hline & 4.75 & 4.66 \\
\hline & 980 & 976 \\
\hline & 4.40 & 4.22 \\
\hline & 2.00 & 1.80 \\
\hline & 1.00 & 0.98 \\
\hline & 9.40 & 8.88 \\
\hline & 10.00 & 9.63 \\
\hline & 5.80 & 5.84 \\
\hline
\end{tabular}


Yield of tomato crop irrigated with untreated industrial sewage effluent and remediated with potassium silicate and compost applications

Table 2. Heavy-metal analysis in soil and irrigation water during the two seasons of the study

\begin{tabular}{|c|c|c|c|c|c|c|c|c|c|}
\hline \multirow{3}{*}{ Soil } & \multirow{2}{*}{ Season } & \multicolumn{10}{|c|}{ Ppm } \\
\cline { 2 - 10 } & & $\mathbf{N i}$ & $\mathbf{P b}$ & $\mathbf{C r}$ & $\mathbf{C d}$ & $\mathbf{F e}$ & $\mathbf{Z n}$ & $\mathbf{M n}$ & $\mathbf{C u}$ \\
\cline { 2 - 10 } & 2017 & 0.475 & 0.414 & 0.152 & 0.003 & 3.880 & 1.620 & 7.480 & 1.770 \\
\cline { 2 - 11 } & 2018 & 0.483 & 0.421 & 0.163 & 0.005 & 3.952 & 1.747 & 7.634 & 1.810 \\
\hline Irrigation & 2017 & 0.222 & 0.010 & 0.00 & 0.068 & 9.350 & 1.805 & 0.539 & 0.671 \\
water & 2018 & 0.123 & 0.21 & 0.00 & 0.071 & 9.342 & 1.818 & 0.547 & 0.683 \\
\hline
\end{tabular}

All standard agricultural practices other than experimental treatments were applied according to the recommendations of the ministry of Agriculture, Egypt.

\subsection{Experimental treatments}

During the preparation of the soil and before transplanting, four levels of compost were applied namely, 0 (control), 10, 20, and $30 \mathrm{~m}^{3} / \mathrm{feddan}$ and mixed well with the upper $50 \mathrm{~cm}$ of soil. A complete analysis of the applied compost is shown in Table 3.

Potassium silicate $\left(\mathrm{SiO}_{2} 25 \%-\mathrm{K}_{2} \mathrm{O} 15 \%\right)$ were sprayed on the plants three times starting at 20 days after transplanting and with 20 days interval. Four spraying concentrations were applied namely 0.0 (control), 3.0, 4.0 and $5.0 \mathrm{~cm}^{3} / 1$.

Table 3. Analysis of compost during the two seasons of the study

\begin{tabular}{|l|c|c|}
\hline \multicolumn{1}{|c|}{ Analysis } & Unit & Pure Compost \\
\hline Weight of $1 \mathrm{~m}^{3}$ & $\mathrm{Kg}$ & 653 \\
Moisture & $(\%)$ & 34 \\
$\mathrm{pH}\left(\mathrm{H}_{2} \mathrm{O}\right) 1: 10$ & - & 6,16 \\
$\mathrm{EC}\left(\mathrm{dsm}^{-1}\right) 1: 10$ & $\mathrm{Ds} / \mathrm{m}$ & 4,14 \\
Total nitrogen & $(\%)$ & 1,36 \\
Ammonium & $\mathrm{Ppm}$ & 76 \\
nitrogen $\left(\mathrm{NH}_{4}\right)$ & & \\
Nitrate & $\mathrm{Ppm}$ & 57 \\
nitrogen( $\left.\mathrm{NO}_{3}\right)$ & & 39,13 \\
Organic matter & $(\%)$ & 22,87 \\
Total carbon & $(\%)$ & 60,57 \\
Ash & $(\%)$ & 1,66 \\
C/N ratio & - & 0,59 \\
Total phosphorus & $(\%)$ & 1,32 \\
Total potassium & $(\%)$ & - \\
\hline Nematodes & - & $-\mathrm{ve}$ \\
Cause diseases to & Larva/200g & \\
plant & & $-\mathrm{ve}$ \\
Free not cause & Larva/200g & \\
diseases & & \\
\hline
\end{tabular}

\subsection{Measurements}

Plant destructed samples were taken in the end of the seasons to determine plant height, and number of leaves and branches. Total yield/plant was measured by the end of the season when all ripe fruits were harvested. $\mathrm{Zn}, \mathrm{Mn}, \mathrm{Pb}, \mathrm{Ni}$ and $\mathrm{Cd}$ contents were determined with a Model SOLAR 969 Atomic Absorption Spectrometer (FAO/WHO, 2001). SPAD readings at 90 days after transplanting was measured in fully expanded leaves using Minolta SPAD 501 chlorophyll meter.

\subsection{Experimental design and statistical analysis}

The treatments were arranged in a split plot design with four replicates where potassium silicate treatments were in the main plot and compost treatments in the sub main plots. All data collected were subjected to the statistical analysis according to Snedecor and Cochram (1968). The data of treatments were compared, using least significant difference (LSD) method at 0.05 as mentioned by Gomez and Gomez (1984).

\section{Results}

Data in Table 4 show that potassium silicate spraying increased significantly plant length and number of leaves and branches per plant as the concentration of the application increased in both growing seasons.

As for the effect of compost adding, compost application showed a gradual positive effect on plant length, and number of leaves and branches per plant with the highest effect recorded with the highest rate of application (30 m $/ \mathrm{fed}$.) Table 4.

The interaction effect of potassium silicate and compost showed an added effect of both treatments on all measured parameters. The highest concentration of potassium silicate with the highest rate of compost gave the tallest plants and highest number of leaves and branches. 
Table 4. Effect of foliar application of potassium silicate, compost adding and their interaction on plant length, and number of leaves and branches of tomato plants in 2017 and 2018 seasons

\begin{tabular}{|c|c|c|c|c|c|c|c|}
\hline \multirow{2}{*}{\multicolumn{2}{|c|}{ Treatments }} & \multicolumn{2}{|c|}{ Seasons } & \multicolumn{2}{|c|}{ Seasons } & \multicolumn{2}{|c|}{ Seasons } \\
\hline & & 2017 & 2018 & 2017 & 2018 & 2017 & 2018 \\
\hline \multicolumn{2}{|c|}{ Potassium silicate } & \multicolumn{2}{|c|}{ Plant length (cm) } & \multicolumn{2}{|c|}{ Leaves No. } & \multicolumn{2}{|c|}{ BranchesNo } \\
\hline & $0 \mathrm{~cm}^{3} / \mathrm{L}$ & 77.55 & 75.85 & 66.78 & 69.84 & 9.86 & 11.01 \\
\hline & $3 \mathrm{~cm}^{3} / \mathrm{L}$ & 86.37 & 84.19 & 74.38 & 77.08 & 10.74 & 11.66 \\
\hline & $4 \mathrm{~cm}^{3} / \mathrm{L}$ & 96.79 & 94.72 & 81.03 & 83.72 & 11.27 & 12.06 \\
\hline & $5 \mathrm{~cm}^{3} / \mathrm{L}$ & 101.52 & 99.24 & 86.19 & 89.13 & 11.87 & 12.85 \\
\hline \multicolumn{2}{|c|}{ L.S.D at $5 \%$ level } & 2.13 & 1.98 & 2.93 & 3.16 & 0.62 & 0.44 \\
\hline \multicolumn{8}{|c|}{ Compost } \\
\hline \multirow{4}{*}{\multicolumn{2}{|c|}{$\begin{array}{c}0 \mathrm{~m}^{3} / \text { fed. } \\
10 \mathrm{~m}^{3} / \text { fed. } \\
20 \mathrm{~m}^{3} / \text { fed. } \\
30 \mathrm{~m}^{3} / \text { fed. }\end{array}$}} & 85.96 & 83.78 & 65.32 & 70.36 & 9.28 & 10.61 \\
\hline & & 88.58 & 86.77 & 73.90 & 76.56 & 10.21 & 10.70 \\
\hline & & 91.85 & 90.06 & 79.96 & 82.69 & 11.49 & 12.45 \\
\hline & & 95.85 & 93.33 & 86.92 & 89.91 & 12.73 & 13.81 \\
\hline \multicolumn{2}{|c|}{ L.S.D at $5 \%$ level } & 1.44 & 1.32 & 3.34 & 3.57 & 0.59 & 0.63 \\
\hline \multicolumn{8}{|c|}{ Interaction } \\
\hline \multirow{4}{*}{$0 \mathrm{~cm}^{3} / \mathrm{L}$} & $0 \mathrm{~m}^{3}$ Compost / fed. & 70.45 & 68.54 & 54.23 & 57.32 & 8.12 & 9.65 \\
\hline & $10 \mathrm{~m}^{3}$ Compost / fed. & 74.23 & 72.56 & 61.56 & 64.22 & 9.65 & 10.65 \\
\hline & $20 \mathrm{~m}^{3}$ Compost / fed. & 80.22 & 78.76 & 70.44 & 73.41 & 10.22 & 11.22 \\
\hline & $30 \mathrm{~m}^{3}$ Compost / fed. & 85.33 & 83.54 & 80.81 & 84.45 & 11.45 & 12.55 \\
\hline \multirow{4}{*}{$3 \mathrm{~cm}^{3} / \mathrm{L}$} & $0 \mathrm{~m}^{3}$ Compost / fed. & 81.97 & 79.43 & 62.68 & 65.71 & 9.34 & 10.11 \\
\hline & $10 \mathrm{~m}^{3}$ Compost / fed. & 84.45 & 82.66 & 71.65 & 74.29 & 9.98 & 10.85 \\
\hline & $20 \mathrm{~m}^{3}$ Compost / fed. & 86.49 & 84.56 & 78.56 & 81.11 & 11.43 & 12.38 \\
\hline & $30 \mathrm{~m}^{3}$ Compost / fed. & 92.59 & 90.11 & 84.66 & 87.23 & 12.22 & 13.32 \\
\hline \multirow{4}{*}{$4 \mathrm{~cm}^{3} / \mathrm{L}$} & $0 \mathrm{~m}^{3}$ Compost / fed. & 93.23 & 91.22 & 73.84 & 76.11 & 9.67 & 10.92 \\
\hline & $10 \mathrm{~m}^{3}$ Compost / fed. & 95.55 & 93.71 & 78.44 & 81.43 & 10.20 & 10.01 \\
\hline & $20 \mathrm{~m}^{3}$ Compost / fed. & 98.45 & 96.65 & 82.43 & 85.66 & 11.89 & 12.92 \\
\hline & $30 \mathrm{~m}^{3}$ Compost / fed. & 99.94 & 97.33 & 89.42 & 91.71 & 13.33 & 14.41 \\
\hline \multirow{4}{*}{$5 \mathrm{~cm}^{3} / \mathrm{L}$} & $0 \mathrm{~m}^{3}$ Compost / fed. & 98.21 & 96.11 & 79.56 & 82.32 & 10.11 & 11.76 \\
\hline & $10 \mathrm{~m}^{3}$ Compost / fed. & 100.01 & 98.17 & 83.98 & 86.33 & 10.99 & 10.99 \\
\hline & $20 \mathrm{~m}^{3}$ Compost / fed. & 102.22 & 100.30 & 88.42 & 91.67 & 12.45 & 13.67 \\
\hline & $30 \mathrm{~m}^{3}$ Compost / fed. & 105.56 & 102.37 & 92.82 & 96.28 & 13.93 & 14.98 \\
\hline \multicolumn{2}{|c|}{ L.S.D at $5 \%$ level } & 2.01 & 1.91 & 2.53 & 2.76 & 1.32 & 1.68 \\
\hline
\end{tabular}

Data in Table 5 indicate that potassium silicate spraying increased significantly SPAD reading and total yield per plant as the concentration of the application increased in both growing seasons.

As for the effect of compost adding, compost application showed a gradual positive effect on SPAD reading and total yield per plant with the highest effect recorded with the highest rate of application (30 $\mathrm{m}^{3} / \mathrm{fed}$.) Table 5.

The interaction effect of potassium silicate and compost showed an added effect of both treatments on all measured parameters. The highest concentration of potassium silicate with the highest rate of compost gave the highest SPAD reading and total yield per plant.
Data in Table 6 indicated that $\mathrm{Zn}$ and $\mathrm{Mn}$ contents responded negatively and significantly to the increment in potassium silicate application rates Table 6.

Also, increasing compost application rate significantly decreased the contents of $\mathrm{Zn}$ and $\mathrm{Mn}$ in the tissue of the plants with the highest negative effect recorded with the highest rate of application (30 $\mathrm{m}^{3} / \mathrm{fed}$.) in two seasons of study Table 6 .

Data in Table 6 showed $\mathrm{Zn}$ and $\mathrm{Mn}$ contents responded negatively and significantly to the interaction effect of the treatments showing the highest effect with the treatment $5 \mathrm{~cm}^{3}$ potassium silicate associated with $30 \mathrm{~m}^{3}$ compost application compared to control. 

and remediated with potassium silicate and compost applications

Table 5. Effect of foliar application of potassium silicate, compost adding and their interaction on SPAD readings and total yield of tomato plants in 2017 and 2018 seasons

\begin{tabular}{|c|c|c|c|c|c|}
\hline \multirow{2}{*}{\multicolumn{2}{|c|}{ Treatments }} & \multicolumn{2}{|c|}{ Seasons } & \multicolumn{2}{|c|}{ Seasons } \\
\hline & & 2017 & 2018 & 2017 & 2018 \\
\hline \multicolumn{2}{|c|}{ Potassium silicate } & \multicolumn{2}{|c|}{ SPAD readings } & \multicolumn{2}{|c|}{ Total yield/plant $(\mathrm{g})$} \\
\hline \multicolumn{2}{|c|}{$0 \mathrm{~cm}^{3} / \mathrm{L}$} & 38.44 & 39.41 & 2145.18 & 2258.11 \\
\hline \multicolumn{2}{|c|}{$3 \mathrm{~cm}^{3} / \mathrm{L}$} & 41.39 & 42.65 & 2421.58 & 2470.37 \\
\hline \multicolumn{2}{|c|}{$4 \mathrm{~cm}^{3} / \mathrm{L}$} & 45.83 & 46.83 & 2798.49 & 2897.84 \\
\hline \multicolumn{2}{|c|}{$5 \mathrm{~cm}^{3} / \mathrm{L}$} & 48.20 & 49.13 & 3073.94 & 3155.67 \\
\hline \multicolumn{2}{|c|}{ L.S.D at $5 \%$ level } & 1.33 & 1.42 & 23.57 & 29.39 \\
\hline \multicolumn{6}{|c|}{ Compost } \\
\hline \multicolumn{2}{|c|}{$0 \mathrm{~m}^{3} / \mathrm{fed}$} & 38.40 & 39.47 & 2214.46 & 2258.74 \\
\hline \multicolumn{2}{|c|}{$10 \mathrm{~m}^{3} /$ fed } & 41.02 & 41.73 & 2441.76 & 2554.98 \\
\hline \multicolumn{2}{|c|}{$20 \mathrm{~m}^{3} /$ fed } & 45.10 & 46.10 & 2716.40 & 2807.81 \\
\hline \multicolumn{2}{|c|}{$30 \mathrm{~m}^{3} / \mathrm{fed}$} & 49.72 & 50.73 & 3066.65 & 3144.87 \\
\hline \multicolumn{2}{|c|}{ L.S.D at $5 \%$ level } & 1.50 & 1.64 & 27.58 & 34.32 \\
\hline \multicolumn{6}{|c|}{ Interaction } \\
\hline \multirow{4}{*}{$0 \mathrm{~cm}^{3} / \mathrm{L}$} & $0 \mathrm{~m}^{3}$ Compost / fed. & 30.82 & 31.45 & 1654.32 & 1756.21 \\
\hline & $10 \mathrm{~m}^{3}$ Compost / fed. & 33.67 & 34.54 & 1956.64 & 2107.72 \\
\hline & $20 \mathrm{~m}^{3}$ Compost / fed. & 41.88 & 42.91 & 2285.43 & 2378.53 \\
\hline & $30 \mathrm{~m}^{3}$ Compost / fed. & 47.41 & 48.76 & 2684.34 & 2790.63 \\
\hline \multirow{4}{*}{$3 \mathrm{~cm}^{3} / \mathrm{L}$} & $0 \mathrm{~m}^{3}$ Compost / fed. & 35.15 & 36.55 & 1967.87 & 1854.79 \\
\hline & $10 \mathrm{~m}^{3}$ Compost / fed. & 38.83 & 39.27 & 2269.91 & 2372.89 \\
\hline & $20 \mathrm{~m}^{3}$ Compost / fed. & 43.54 & 44.72 & 2554.11 & 2672.31 \\
\hline & $30 \mathrm{~m}^{3}$ Compost / fed. & 49.48 & 50.09 & 2895.34 & 2981.51 \\
\hline \multirow{4}{*}{$4 \mathrm{~cm}^{3} / \mathrm{L}$} & $0 \mathrm{~m}^{3}$ Compost / fed. & 42.36 & 43.56 & 2493.34 & 2572.44 \\
\hline & $10 \mathrm{~m}^{3}$ Compost / fed. & 43.89 & 44.78 & 2656.53 & 2773.61 \\
\hline & $20 \mathrm{~m}^{3}$ Compost / fed. & 46.72 & 47.44 & 2848.71 & 2961.68 \\
\hline & $30 \mathrm{~m}^{3}$ Compost / fed. & 50.63 & 51.56 & 3195.39 & 3283.63 \\
\hline \multirow{4}{*}{$5 \mathrm{~cm}^{3} / \mathrm{L}$} & $0 \mathrm{~m}^{3}$ Compost / fed. & 45.25 & 46.34 & 2742.32 & 2851.52 \\
\hline & $10 \mathrm{~m}^{3}$ Compost / fed. & 47.67 & 48.34 & 2884.52 & 2965.72 \\
\hline & $20 \mathrm{~m}^{3}$ Compost / fed. & 48.54 & 49.34 & 3177.38 & 3281.74 \\
\hline & $30 \mathrm{~m}^{3}$ Compost / fed. & 51.34 & 52.52 & 3491.56 & 3523.71 \\
\hline \multicolumn{2}{|c|}{ L.S.D at $5 \%$ level } & 2.67 & 2.51 & 33.67 & 45.42 \\
\hline
\end{tabular}


Table 6. Effect of foliar application of potassium silicate, compost adding and their interaction on $\mathrm{Zn}$ and $\mathrm{Mn}$ content of tomato plants in 2017 and 2018 seasons

\begin{tabular}{|c|c|c|c|c|c|}
\hline \multirow{2}{*}{\multicolumn{2}{|c|}{ Treatments }} & \multicolumn{2}{|c|}{ Seasons } & \multicolumn{2}{|c|}{ Seasons } \\
\hline & & 2017 & 2018 & 2017 & 2018 \\
\hline \multicolumn{2}{|c|}{ Potassium silicate } & \multicolumn{2}{|c|}{ Zncontent } & \multicolumn{2}{|c|}{ Mn content } \\
\hline \multicolumn{2}{|c|}{$0 \mathrm{~cm}^{3} / \mathrm{L}$} & 40.73 & 43.46 & 11.30 & 12.02 \\
\hline \multicolumn{2}{|c|}{$3 \mathrm{~cm}^{3} / \mathrm{L}$} & 37.21 & 39.72 & 11.04 & 11.62 \\
\hline \multicolumn{2}{|c|}{$4 \mathrm{~cm}^{3} / \mathrm{L}$} & 33.18 & 35.72 & 10.50 & 11.00 \\
\hline \multicolumn{2}{|c|}{$5 \mathrm{~cm}^{3} / \mathrm{L}$} & 27.99 & 30.74 & 10.02 & 10.62 \\
\hline \multicolumn{2}{|c|}{ L.S.D at $5 \%$ level } & 2.11 & 2.91 & 0.85 & 0.91 \\
\hline \multicolumn{6}{|c|}{ Compost } \\
\hline \multicolumn{2}{|c|}{$0 \mathrm{~m}^{3} / \mathrm{fed}$} & 40.73 & 45.84 & 11.31 & 12.09 \\
\hline \multicolumn{2}{|c|}{$10 \mathrm{~m}^{3} /$ fed. } & 36.16 & 38.97 & 11.03 & 11.76 \\
\hline \multicolumn{2}{|c|}{$20 \mathrm{~m}^{3} /$ fed } & 32.54 & 35.41 & 10.64 & 11.32 \\
\hline \multicolumn{2}{|c|}{$30 \mathrm{~m}^{3} / \mathrm{fed}$} & 27.22 & 29.41 & 10.02 & 10.08 \\
\hline \multicolumn{2}{|c|}{ L.S.D at $5 \%$ level } & 3.23 & 3.71 & 0.88 & 0.90 \\
\hline \multicolumn{6}{|c|}{ Interaction } \\
\hline \multirow{4}{*}{$0 \mathrm{~cm}^{3} / \mathrm{L}$} & $0 \mathrm{~m}^{3}$ Compost / fed. & 50.78 & 53.11 & 11.89 & 12.93 \\
\hline & $10 \mathrm{~m}^{3}$ Compost / fed. & 41.43 & 44.09 & 11.41 & 12.50 \\
\hline & $20 \mathrm{~m}^{3}$ Compost / fed. & 38.29 & 41.33 & 11.15 & 11.85 \\
\hline & $30 \mathrm{~m}^{3}$ Compost / fed. & 32.44 & 35.32 & 10.71 & 10.83 \\
\hline \multirow{4}{*}{$3 \mathrm{~cm}^{3} / \mathrm{L}$} & $0 \mathrm{~m}^{3}$ Compost / fed. & 45.75 & 48.24 & 11.65 & 12.41 \\
\hline & $10 \mathrm{~m}^{3}$ Compost / fed. & 39.59 & 42.12 & 11.42 & 12.11 \\
\hline & $20 \mathrm{~m}^{3}$ Compost / fed. & 34.39 & 37.91 & 10.81 & 11.62 \\
\hline & $30 \mathrm{~m}^{3}$ Compost / fed. & 29.12 & 30.61 & 10.30 & 10.36 \\
\hline \multirow{4}{*}{$4 \mathrm{~cm}^{3} / \mathrm{L}$} & $0 \mathrm{~m}^{3}$ Compost / fed. & 42.47 & 45.35 & 10.91 & 11.62 \\
\hline & $10 \mathrm{~m}^{3}$ Compost / fed. & 33.56 & 36.29 & 10.77 & 11.39 \\
\hline & $20 \mathrm{~m}^{3}$ Compost / fed. & 30.27 & 33.03 & 10.50 & 11.07 \\
\hline & $30 \mathrm{~m}^{3}$ Compost / fed. & 26.13 & 28.22 & 9.71 & 9.94 \\
\hline \multirow{4}{*}{$5 \mathrm{~cm}^{3} / \mathrm{L}$} & $0 \mathrm{~m}^{3}$ Compost / fed. & 33.45 & 36.67 & 10.80 & 11.41 \\
\hline & $10 \mathrm{~m}^{3}$ Compost / fed. & 30.09 & 33.41 & 10.52 & 11.12 \\
\hline & $20 \mathrm{~m}^{3}$ Compost / fed. & 27.22 & 29.40 & 10.13 & 10.74 \\
\hline & $30 \mathrm{~m}^{3}$ Compost / fed. & 21.21 & 23.51 & 9.37 & 9.22 \\
\hline \multicolumn{2}{|c|}{ L.S.D at $5 \%$ level } & 5.56 & 5.23 & 1.02 & 1.11 \\
\hline
\end{tabular}

Data in Table 7 indicated that $\mathrm{Pb}, \mathrm{Ni}$ and $\mathrm{Cd}$ contents responded negatively and significantly to the increment in potassium silicate application rates Table 7.

Also, increasing compost application rate significantly decreased the contents of $\mathrm{Pb}, \mathrm{Ni}$ and $\mathrm{Cd}$ in the tissue of the plants in two seasons of study Table 7
Data in Table 7 showed $\mathrm{Pb}, \mathrm{Ni}$ and $\mathrm{Cd}$ contents responded negatively and significantly to the interaction effect of the treatments showing the highest effect with the treatment $5 \mathrm{~cm}^{3}$ potassium silicate associated with $30 \mathrm{~m}^{3}$ compost application compared to control. 
Yield of tomato crop irrigated with untreated industrial sewage effluent and remediated with potassium silicate and compost applications

Table 7. Effect of foliar application of potassium silicate, compost adding and their interaction on $\mathrm{Pb}, \mathrm{Ni}$ and Cd contents of tomato plants in 2017 and 2018 seasons

\begin{tabular}{|c|c|c|c|c|c|c|c|}
\hline \multirow{2}{*}{\multicolumn{2}{|c|}{ Treatments }} & \multicolumn{2}{|c|}{ Seasons } & \multicolumn{2}{|c|}{ Seasons } & \multicolumn{2}{|c|}{ Seasons } \\
\hline & & 2017 & 2018 & 2017 & 2018 & 2017 & 2018 \\
\hline \multicolumn{2}{|c|}{ Potassium silicate } & \multicolumn{2}{|c|}{ Pbcontent } & \multicolumn{2}{|c|}{ Ni content (ppm) } & \multicolumn{2}{|c|}{ Cd content (ppm) } \\
\hline & $0 \mathrm{~cm}^{3} / \mathrm{L}$ & 31.13 & 33.82 & 8.31 & 8.53 & 8.81 & 7.78 \\
\hline & $3 \mathrm{~cm}^{3} / \mathrm{L}$ & 25.61 & 27.84 & 6.20 & 5.98 & 6.51 & 5.50 \\
\hline & $4 \mathrm{~cm}^{3} / \mathrm{L}$ & 18.95 & 21.54 & 4.06 & 3.45 & 4.85 & 4.10 \\
\hline & $5 \mathrm{~cm}^{3} / \mathrm{L}$ & 12.26 & 18.09 & 3.03 & 2.81 & 3.84 & 2.95 \\
\hline \multicolumn{2}{|c|}{ L.S.D at $5 \%$ level } & 2.39 & 2.92 & 0.53 & 0.47 & 1.45 & 1.11 \\
\hline \multicolumn{8}{|c|}{ Compost } \\
\hline \multirow{4}{*}{\multicolumn{2}{|c|}{$\begin{array}{c}0 \mathrm{~m}^{3} / \text { fed. } \\
10 \mathrm{~m}^{3} / \text { fed. } \\
20 \mathrm{~m}^{3} / \text { fed. } \\
30 \mathrm{~m}^{3} / \text { fed. }\end{array}$}} & 28.76 & 30.77 & 7.58 & 6.59 & 8.65 & 7.53 \\
\hline & & 24.54 & 27.17 & 6.05 & 5.43 & 6.95 & 5.99 \\
\hline & & 21.24 & 24.13 & 4.42 & 4.63 & 4.72 & 3.25 \\
\hline & & 16.40 & 19.21 & 3.55 & 4.12 & 3.70 & 3.07 \\
\hline \multicolumn{2}{|c|}{ L.S.D at $5 \%$ level } & 3.01 & 3.22 & 0.43 & 0.51 & 1.22 & 1.19 \\
\hline \multicolumn{8}{|c|}{ Interaction } \\
\hline \multirow{4}{*}{$0 \mathrm{~cm}^{3} / \mathrm{L}$} & $0 \mathrm{~m}^{3}$ Compost / fed. & 40.16 & 42.21 & 12.42 & 11.56 & 12.45 & 11.21 \\
\hline & $10 \mathrm{~m}^{3}$ Compost / fed. & 32.05 & 34.31 & 9.33 & 8.32 & 10.22 & 9.11 \\
\hline & $20 \mathrm{~m}^{3}$ Compost / fed. & 29.20 & 32.32 & 6.54 & 7.67 & 7.11 & 6.23 \\
\hline & $30 \mathrm{~m}^{3}$ Compost / fed. & 23.10 & 26.43 & 4.94 & 6.56 & 5.45 & 4.56 \\
\hline \multirow{4}{*}{$3 \mathrm{~cm}^{3} / \mathrm{L}$} & $0 \mathrm{~m}^{3}$ Compost / fed. & 31.92 & 33.61 & 8.61 & 7.45 & 9.56 & 8.45 \\
\hline & $10 \mathrm{~m}^{3}$ Compost / fed. & 28.20 & 30.12 & 7.52 & 6.84 & 7.78 & 6.81 \\
\hline & $20 \mathrm{~m}^{3}$ Compost / fed. & 23.10 & 25.42 & 5.11 & 5.11 & 4.94 & 3.75 \\
\hline & $30 \mathrm{~m}^{3}$ Compost / fed. & 19.20 & 22.22 & 3.55 & 4.52 & 3.74 & 2.99 \\
\hline \multirow{4}{*}{$4 \mathrm{~cm}^{3} / \mathrm{L}$} & $0 \mathrm{~m}^{3}$ Compost / fed. & 23.52 & 25.91 & 5.73 & 4.22 & 7.34 & 6.33 \\
\hline & $10 \mathrm{~m}^{3}$ Compost / fed. & 20.60 & 23.34 & 4.32 & 3.65 & 5.33 & 4.56 \\
\hline & $20 \mathrm{~m}^{3}$ Compost / fed. & 18.55 & 21.46 & 3.21 & 3.01 & 3.82 & 2.79 \\
\hline & $30 \mathrm{~m}^{3}$ Compost / fed. & 13.11 & 15.43 & 2.99 & 2.90 & 2.92 & 2.71 \\
\hline \multirow{4}{*}{$5 \mathrm{~cm}^{3} / \mathrm{L}$} & $0 \mathrm{~m}^{3}$ Compost / fed. & 19.42 & 21.34 & 3.57 & 3.11 & 5.23 & 4.11 \\
\hline & $10 \mathrm{~m}^{3}$ Compost / fed. & 17.30 & 20.92 & 3.01 & 2.89 & 4.45 & 3.45 \\
\hline & $20 \mathrm{~m}^{3}$ Compost / fed. & 14.12 & 17.33 & 2.82 & 2.71 & 3.01 & 2.21 \\
\hline & $30 \mathrm{~m}^{3}$ Compost / fed. & 10.20 & 12.76 & 2.72 & 2.51 & 2.67 & 2.03 \\
\hline \multicolumn{2}{|r|}{ L.S.D at $5 \%$ level } & 5.19 & 6.34 & 1.03 & 0.9 & 1.02 & 0.86 \\
\hline
\end{tabular}

\section{Discussion}

Heavy metals have been reported to cause deteriorating effects on different plant growth aspects resulting in reduction in plant production and quality. $\mathrm{Cd}, \mathrm{Ni}$ and $\mathrm{Pb}$ are such example of those heavy metals exist in agricultural soils because of misuse of agrochemicals and/or pollution from other sources such as irrigation of raw untreated sewage effluent. In this study, the deteriorating effect of heavy metal polluted irrigation water has been observed leading to the lowest growth and production of tomato plants as well as the highest contents of heavy metals in plant tissue. Such accumulation has been observed earlier in many crops (Nagajyoti et al 2010). In this study, compost application reduced the harmful effect of heavy metals existed in irrigation water. This can be due to changing the physicochemical property of soils and reacting with heavy metals (Bolan et al 2014, Liu et al 2009). The beneficial effect of compost application in reducing the harmful effects of heavy metals has been reported earlier by Huang et al (2016). On the other hand, potassium silicate showed also an ameliorating effect on plant growth and production as revealed from our data. These positive effects are 
mainly due to the presence of $\mathrm{Si}$. These beneficial effects have been explained on the basis that $\mathrm{Si}$ increase plant resistance to some heavy metals such as $\mathrm{Cd}$ by inhibiting $\mathrm{Cd}$ uptake in roots and the enhancement of light-use-efficiency in leaves (Nwugo and Huerta $2008 \mathrm{a \& b}$ ). Silicon has been reported to have a beneficial effect on growth and yield for various horticultural plant species such as bean, cucumber (Zhu et al 2004), tomato (Romero- Aranda et al 2006) and Zucchini squash (Savvas et al 2015).

Because of the different mode of action for compost and potassium silicate against heavy metals, the interaction of the two treatments was additive and its combination resulted in enhancing the effect of each other which reflected on higher plant growth and production.

\section{Conclusion}

It can be concluded that the treatment with compost and potassium silicate, as well as the interaction between them, reduced the harmful effect of irrigation with untreated industrial wastewater, as well as increased the vegetative growth and yield of the tomato plants under study.

\section{References}

Abdel-Mawgoud, AMR (2005) Growth, yield and quality of green bean (Phaseolus vulgaris) in response to irrigation and compost applications. J of Applied Sci Research 2, 443-450.

Abdel-Mawgoud, AMR; El-Nemr, MA; Tanatawy, AS; Habib, HA (2010) Alleviation of salinity effects on green bean plants using some environmental friendly materials. J Appl Sci Res 6, 871-878.

Arao, T; Ishikawa, S; Murakami, M; Abe, K; Maejimaand, Y; Makino, T (2010) Heavymetal contamination of agricultural soil and countermeasures in Japan. Paddy and Water Environment 8, 247-257.

Bolan, N; Kunhikrishnan, A; Thangarajan, R; Kumpiene, J; Park, J; Makino, T (2014) Remediation of heavy metal (loid)s contaminated soils to mobilize or to imitedmobilize. J of Hazardous Materials 266, 141-166.

De la Fuente, C; Clemente, R; Martinez-Alcala, I; Tortosa, G; Pilar Bernal, M (2011) Impact of fresh and composted solid olive husk and their water-soluble fractions on soil heavy metal fractionation; microbial biomass and plant uptake. $J$ of Hazardous Materials 186, 1283-1289.
Emamverdian, A; Ding, Y; Xie, Y; Sangari, S (2018) Silicon Mechanisms to Ameliorate Heavy Metal Stress in Plants. Hindawi, Bio Med Research International. https://doi.org/10.1155/2018/8492898 10 p.

Epstein, E (1999) Silicon Annu Rev Plant Physiol Mol Boil 50, 641-664.

FAO/WHO (2001) "Food additives contaminants" joint codex Alimentarius commission, Food and Agriculture Organization of the United Nations /world health Organization (FAO/WHO), Food Standards program ALINORM01/12A.

Farooq, MA; Ali, S; Hameed, A; Ishaque, W; Mahmood, K; Iqbal, Z (2013) Alleviation of cadmium toxicity by silicon is related to elevated photosynthesis, antioxidant enzymes; suppressed cadmium uptake and oxidative stress in cotton. Ecotoxicol Environ Saf 96, 242-249.

Gomez, KA; Gomez, AA (1984) Statistical procedures for agriculture research. Second Ed. WielyInter Science Publ. John Wiley \& Sons, New York, USA.

Gu, HH; Zhan, SS; Wang, SZ; Tang, YT; Chaney, RL; Fang, XH; Cai, XD; Qiu, RL (2012) Silicon-mediated amelioration of zinc toxicity in rice (Oryze sativa L.) seedlings. Plant Soil 350, 193-204.

He, Z; Zhang, M; Calvert, D; Stoffella, P; Yang, X; Yu, S (2004) Transport of heavy metals in surface runoff from vegetable and citrus fields. Soil Sci Society of America J 68, 1662-1669.

Huang, M; Zhu, Y; Li, Z; Huang, B; Luo, N; Liu, C; Zeng, G (2016) Compost as a Soil Amendment to Remediate Heavy Metal-Contaminated Agricultural Soil: Mechanisms, Efficacy, Problems, and Strategies. Water Air Soil Pollut 227, 359.

Huang, DY; Zhuang, L; Cao, WD; Xu, W; Zhou, SG; $\mathrm{Li}, \mathrm{FB}$ (2010) Comparison of dissolved organic matter from sewage sludge and sludge compost as electron shuttles for enhancing Fe (III) bio reduction. $J$ of Soils and Sediments 10, 722-729.

Hussain, I; Ashraf, MA; Rasheed, R; Asghar, A; Sajid, MA; lqbal, M (2015) Exogenous application of silicon at the boot stage decreases accumulation of cadmium in wheat (Triticumaestivum L.) grains. Braz J Bot, 38, 223-234.

Khan, S; Cao, Q; Zheng, YM; Huang, YZ; Zhu, YG (2008) Health risks of heavy metals in contaminated soils and food crops irrigated with wastewater in 

and remediated with potassium silicate and compost applications

Beijing, China. Environmental Pollution 152, 686692.

Lagomarsino, A; Mench, M; Marabottini, R; Pignataro, A; Grego, S; Renella, G (2011) Copper distribution and hydrolase activities in a contaminated soil amended with dolomitic limestone and compost. Ecotoxicology and Environmental Safety 74, 20132019.

Liang, Y; Wong, JWC; Wei, L (2005) Silicon-mediated enhancement of cadmium tolerance in maize (Zea mays L.) grown in cadmium contaminated soil. Chemosphere 58, 475-483.

Liu, L; Chen, H; Cai, P; Liang, W; Huang, Q (2009) Immobilization and phytotoxicity of $\mathrm{Cd}$ in contaminated soil amended with chicken manure compost. $J$ of Hazardous Materials 163, 563-567.

Ma, J; Cai, H; He, C; Zhang, W; Wang, L (2015) A hemicellulose-bound form of silicon inhibits cadmium ion uptake in rice (Oryza sativa) cells. New Phytol 206, 1063-1074.

Ma, JF; Yamaji, N (2006) Silicon uptake and accumulation in higher plants. Trends Plant Sci 11, 392397.

Nagajyoti, P; Lee, K; Sreekanth, T (2010) Heavy metals, occurrence and toxicity for plants: a review. Environmental Chemistry Letters 8, 199-216.

Nwugo, CC; Huerta, AJ (2008무) Silicon-induced cadmium resistance in rice (Oryza sativa). J Plant Nutr Soil Sci 171, 841-848.

Nwugo, CC; Huerta, AJ (2008b) Effect of silicon nutrition on cadmium uptake, growth and photosynthesis of rice plants exposed to low-level cadmium. Plant Soil 311, 73-86.

Paradelo, R; Villada, A; Barral, MT (2011) Reductionof the short-term availability of copper, lead and zinc in a contaminated soil amended with municipal solid waste compost. $J$ of Hazardous Materials 188, 98-104.

Park, JH; Lamb, D; Paneerselvam, P; Choppala, G; Bolan, N; Chung, JW (2011) Role of organic amendments on enhanced bioremediation of heavy metal (loid) contaminated soils. J of Hazardous Materials $185,549-574$.
Rattan, R; Datta, S; Chhonkar, P; Suribabu, K; Singh, A (2005) Long-term impact of irrigation with sewage effluents on heavy metal content in soils, crops and ground water-a case study. Agriculture, Ecosystems \& Environment 109, 310-322.

Ramachandran, V; D'souza, TJ (1998) Plant Uptake of Cadmium, Zinc, and Manganese in Soils Amended with Sewage Sludge and City Compost. Bulletin of Environmental Contamination and Toxicology 61, 347-354.

Romero-Aranda, MR; Jurado, O; Cuartero J. Silicon (2006) Alleviates the deleterious salt effects on tomato plant growth by improving plant water status. J Plant Physiol 163, 847-855.

Savvas, D; Ntatsi, G (2015) Biostimulant activity of silicon in horticulture. Scientia Horticulturae 196, 6681.

Sharma, S; Dhaliwal, SS (2019) Effect of Sewage Sludge and Rice Straw Compost on Yield, Micronutrient Availability and Soil Quality under Rice-Wheat System. Communications in Soil Science and Plant Analysis 50, 1943-1954.

Snedecor, GW; Cochran, WG (1980) Statistical methods. Sixth Edition, lowa State University Press, Ames., lowa, USA.

Udeigwe, TK; Eze, PN; Teboh, JM; Stietiya, MH (2011) Application chemistry, and environmental implications of contaminant-immobilization amendment son agricultural soil and water quality. Environment International 37, 258-267.

Udovic, M; McBride, MB (2012) Influence of compost addition on lead and arsenic bioavailability in reclaimed orchard soil assessed using Porcellioscaber bioaccumulation test. $J$ of Hazardous Materials 205, 144-149.

Vaca-Paulin, R; Esteller-Alberich, MV; LugodelaFuente, J; Zavaleta-Mancera, HA (2006) Effect of sewage sludge or compost on the sorption and distribution of copper and cadmium in soil. Waste Management 26, 71-81.

Zhu, Z; Wei, G; Li, J; Qian, Q; Yu, J (2004) Silicon alleviates salt stress and increases antioxidant enzymes activity in leaves of salt-stressed cucumber (Cucumis sativus L.). Plant Sci 167, 527-533. 


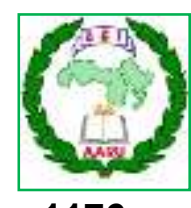

مجلة اتحاد الجامعات العربية للعلوم الزراعية، جامعة عين شمس، القاهرة، مصر

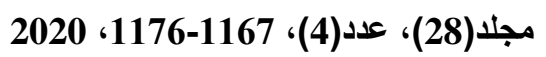

Website: http://ajs.journals.ekb.eg

نمو وإنتاج محصول الطماطم المروية بمياه الصرف الصناعي غير المعالجة ومعالجتها بإستخدام سيليكات البوتاسيوم وسماد الكمبوست النباتي لمباه

[83]

رانه (بو نوفل1" - ايمان علي دياب1 - محمد هاشم2 - - محمد زكى الثناوى2 - هاني جمال عبد الجواد2

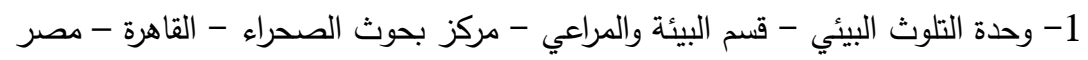
2- قسم البساتين - كلية الزراعة - جامعة عين شمس - ص.ب 68 - حدائق شبرا 11241 - القاهرة - مصر

*Corresponding author: ronyhosny65@gmail.com

Accepted 10 September, 2020

خلال المواسم عند 20 و 40 و 60 يومًا بعد الزرعة

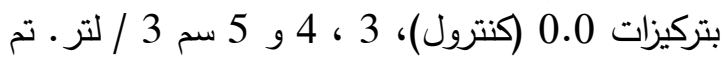
وضع معاملة سيليكات البوتاسيوم في القطع الرئيسية

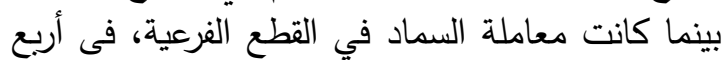

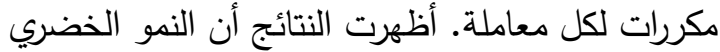

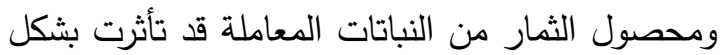
إيجابي من خلال كل من معاملاتسماد الكمبوست النست

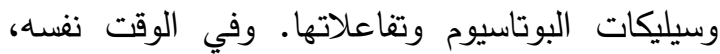

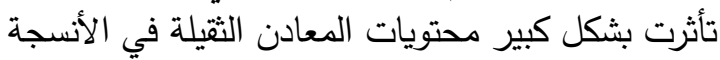
النباتية بهذه المعاملات ـ ويمكن أن نستتتج أن تطبيقات

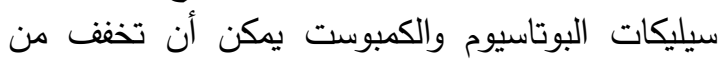
الآثار الضارة للمعادن الثقيلة في التربة.

تم إجراء تجربة في موسمين متتاليين في عامي

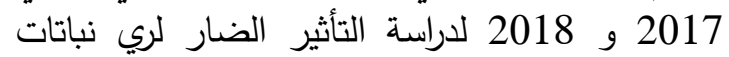

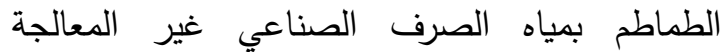

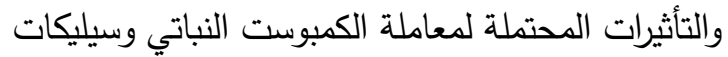

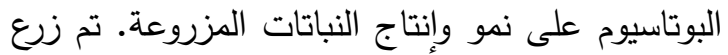

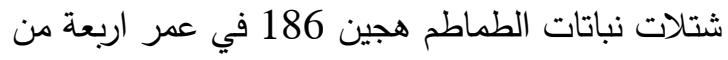

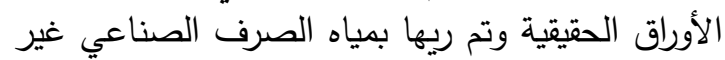
المعالج. تم استخدام الكومبوست أثناء تحضير التربة التباه

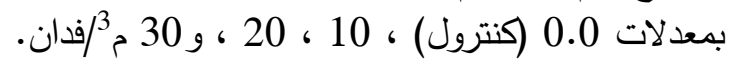
تم رش سيليكات البوتاسيوم على النباتات ثلاث مرات 